Preprints of the

Max Planck Institute for

Research on Collective Goods

Bonn 2010/17

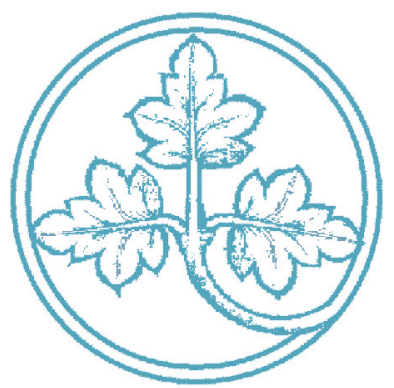

Entry and Incumbent

Innovation

Philipp Weinschenk

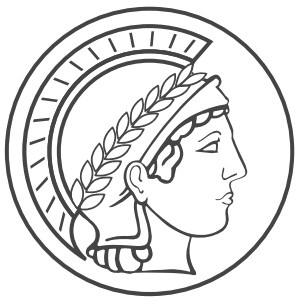

$\overline{\text { MAX PLANCK SOCIETY }}$ 


\section{Entry and Incumbent Innovation}

Philipp Weinschenk

May 2010

Max Planck Institute for Research on Collective Goods, Kurt-Schumacher-Str. 10, D-53113 Bonn http://www.coll.mpg.de 


\title{
ENTRY AND INCUMBENT INNOVATION
}

\author{
Philipp Weinschenk* \\ Bonn Graduate School of Economics \\ and Max Planck Institute for Research on Collective Goods
}

May 5, 2010

\begin{abstract}
We explore how the threat of entry influences the innovation activity of an incumbent. We show that the incumbent's investment is hump-shaped in the entry threat. When the entry threat is small and increases, the incumbent invests more to deter entry, or to make it unlikely. This is due to the entry deterrence effect. However, when the threat becomes huge, entry can no longer profitably be deterred or made unlikely and the investment becomes small. Then the Schumpeterian effect dominates. These results turn out to be very robust.
\end{abstract}

\section{INTRODUCTION}

Even though innovations are central to growth, the question whether more competition or a higher threat of entry leads to greater R\&D investments is not settled. While we do not try to answer this general question, we seek to explore the more specific question how the threat of entry influences an incumbent's investments in R\&D. We build a simple model that captures two important but counteracting effects. First, a Schumpeterian effect. A larger entry threat reduces the incumbent's expected profit and therefore also its investment. Second, an entry deterrence effect. ${ }^{1}$ To deter entry, or to make entry unlikely, a greater threat requires a larger investment.

* Max Planck Institute for Research on Collective Goods, Kurt-Schumacher-Str. 10, 53113 Bonn, Germany, weinschenk@coll.mpg.de. I thank Nadine Bläser, Christoph Engel, Kristoffel Grechenig, Paul Heidhues, Martin Hellwig, Fabian Herweg, Jos Jansen, Daniel Müller, and Susanne Prantl for helpful comments and suggestions.

${ }^{1}$ For the importance of entry in the United States, see Aghion and Howitt (2006, p. 279). Entry deterrence is empirically relevant: "Most R\&D investments made by 
Combining the effects, we find that the incumbent's investment is hump-shaped in the entry threat. Intuitively, when the entry threat is small and increases, the incumbent invests more to deter entry or to make entry unlikely. Then the entry deterrence effect dominates the Schumpeterian effect. However, when the threat becomes huge, entry can no longer profitably be deterred or made unlikely and the investment becomes small. Then the Schumpeterian effect dominates.

There is a discussion in competition policy about the optimal patent breadth, how costly imitation should be, and when antitrust law should require a firm with market power to share its property. ${ }^{2}$ In our model, stronger patent protection/property rights or higher costs of imitation can be interpreted as a weakening of the entry threat. The message of our paper is that a mediocre threat can yield the highest incumbent investments in $\mathrm{R} \& \mathrm{D}$.

Robustness. - Robustness is a central issue in theoretical industrial economics, see Sutton (1990). We therefore explore the robustness of our results in depth.

We show that the hump-shaped relationship between incumbent's R\&D investment and the entry threat is robust to different timings. In the original time structure, the incumbent does not know the rivals' production costs when deciding about investment. Therefore, higher investments only make entry less likely, but entry cannot be deterred for sure in general. In the alternative timing, the incumbent knows the rivals' costs when deciding about investment. Then the incumbent can effectively decide whether to deter entry for sure by investing sufficiently much, or allow entry by investing little or not at all. In both timings, an entry threat may motivate the incumbent to invest more to defend its monopoly. Additionally, zero or small investments become optimal in both timings when the entry threat is overwhelming.

The robustness is remarkable, because it is seldom that fundamental

private firms are aimed at securing market advantage" (Scotchmer 2004, p. 1). See also the empirical study of Goolsbee and Syverson (2008).

${ }^{2}$ See, for example, Gallini (1992), Scotchmer (2004), Segal and Whinston (2007), and Vickers (2009). 
changes of the structure of an extensive form game lead to qualitatively unchanged results. The robustness is important, because the relationship between the intensity of competition and $R \& D$ investment is generally regarded as ambiguous in theoretical models. This is due to the large variety of relevant effects and of the definitions of competition. ${ }^{3}$ We yield clear results by considering two important effects, namely the Schumpeterian and the entry deterrence effect. In Section 5, we explain that our model can be modified in several ways and allows for alternative interpretations of the measure of competition.

Related Literature. - Recently, Aghion et al. (2005b) ${ }^{4}$ and Aghion et al. (2009) applied the concept of step-by-step technological progress to study the relationship between entry and innovation. Their models ${ }^{5}$ are intuitive, but the use of the step-by-step concept in connection with the assumption that entry can only take place at the new technological frontier has two unplausible consequences. ${ }^{6}$ First, an incumbent which is close to the frontier and innovates must not fear entry at all. Second, an incumbent which is further below the frontier cannot prevent entry, no matter how much it invests. We consider a model without step-by-step innovations where the incumbent can choose the size of the innovation and the technology of potential entrants is stochastic. This avoids the aforementioned problems and leads to a richer set of predictions.

In Aghion et al. (2005b, 2009) the influence of a higher threat on a firm's investment is monotone. More specifically, they show that a higher entry threat increases the incumbent's investment when the firm is initially close to the technological frontier, due to the escape-entry effect; it is the other way round if the incumbent is further behind the

\footnotetext{
${ }^{3}$ See, for example, Lee and Wild (1980) vs. Delbono and Denicolo (1991), Gilbert and Sunshine (1995), Belleflamme and Vergari (2006), Sacco and Schmutzler (2007), Schmutzler (2007), Denicolo and Zanchettin (2008), and Vives (2008). For surveys, see Aghion and Griffith (2005) or Gilbert (2006).

${ }^{4} \mathrm{~A}$ companion paper with an emphasis on the empirical aspects is Aghion et al. (2008).

${ }^{5}$ The focus of Aghion et al. (2009) is rather empirically than theoretically.

${ }^{6}$ The problems do not change qualitatively if entrants enter at another, prespecified step.
} 
frontier, due to the discouragement effect. In our model, the relationship is non-monotone because two effects and not only one play a role for a firm in a given situation.

In Aghion et al. (2005a), the interplay between an escape-competition effect and the Schumpeterian effect generates an inverted-U relationship in the aggregate ${ }^{7}$ between $R \& D$ investment and product market competition. They do not consider entry. Instead, they explore the incentives of duopolists to invest in research and development.

Standard models in industrial organization or endogenous growth theory predict that innovation activity declines with competition or entry threat; see Aghion and Griffith (2005). There are several exceptions. Beside the already discussed papers, we want to mention three recent studies. Segal and Whinston (2007) show that in some cases "policies that protect entrants necessarily raise the rate of innovation" (p. 1703). The paper concentrates on innovations made by potential entrants. In Boldrin and Levine (2009), investments in R\&D are higher in a competitive equilibrium than in a monopolistic equilibrium. In Bessen and Maskin's (2009) world of sequential and complementary innovations, no patent protection can be socially desirable because it alleviates imitation, which in turn makes future innovations more likely.

There are two strands of empirical literature to which our paper is related. First, there is a literature on how domestic firms' investments react on the threat of competition by foreign firms. The empirical results are mixed. ${ }^{8}$ Second, the empirical literature on patents (which can be interpreted as barriers to potential entrants) and innovations provides no clear evidence that stronger patents lead to higher investments in innovations. The picture is rather mixed. ${ }^{9}$ This is a puzzling result (Lerner

${ }^{7}$ The influence of more competition on the investment of a certain type of firm is monotone.

${ }^{8}$ See Gilbert and Sunshine (1995), Lelarge and Nefussi (2008), MacDonald (1994), Pavcnik (2002), Javorcik (2004), and Aitken and Harrison (1999). Aghion et al. (2009) find mixed results, in accordance with their theoretical predictions: whether there is a positive or negative effect depends on the distance of the incumbent to the technological frontier.

${ }^{9}$ See the survey of Bessen and Meurer (2008), Mokyr's (2009) study on the role of 
2009, p. 347). In the light of our findings, this is not surprising: even at the firm level, the relationship between an incumbent's investment and the threat of entry is non-monotone.

The paper proceeds as follows. In Section 2, we present the model. In Section 3, we analyze it. The alternative timing is considered in Section 4. Further robustness issues are discussed in Section 5. Section 6 explores the question how important the incumbent's initial production costs are for the relationship between incumbent's R\&D investments and the entry threat. Section 7 concludes. Proofs are in the Appendix.

\section{THE MODEL}

There is an incumbent, firm 0 , and $N$ rivals, firms $1, \ldots, N$. Rivals can enter at cost $Z>0$. They threat the monopoly position of the incumbent. By investing in R\&D the incumbent can lower its production costs. Because the incumbent becomes a stronger competitor, this makes entry for the rivals less attractive. We will explore how the incumbent's optimal investment varies with the quality and the number of rivals.

At Stage 1 , the incumbent chooses its $\mathrm{R} \& \mathrm{D}$ investment $k \geq 0$. The incumbent's per-unit production costs are $c_{0}(k)$. The function $c_{0}$ is twice differentiable and satisfies the following assumptions.

A S S U M P T I O N 1: (i) $c_{0}^{\prime}(k)<0$, (ii) $c_{0}^{\prime \prime}(k)>0$, (iii) $\lim _{k \rightarrow \infty} c_{0}(k)>$ $Z$, (iv) $c_{0}^{\prime}(0)<-1$.

In words, (i) says that a higher investment lowers production costs; (ii) that there are decreasing returns to scale in $\mathrm{R} \& \mathrm{D}$; (iii) that it is not possible to yield production costs that make entry impossible; (iv) that when there is no entry threat, investing at least a tiny amount is optimal, see below. Define $C:=c_{0}(0)$. Note that Parts (i) and (iii) imply $C>Z$.

At Stage 2 , the rivals' per-unit production costs $\left(c_{1}, \ldots, c_{N}\right)$ are drawn. ${ }^{10}$ We will later make concrete assumptions on the distributions.

the patent system in the British Industrial Revolution, or Lerner's (2009) study on the impacts of shifts in patent policy across 60 countries.

${ }^{10} \mathrm{We}$ do not model how theses costs are determined. They can be a result of already made or planned $R \& D$ of the rival firms. 
At Stage 3, the rivals decide upon entry in an arbitrary order, potentially simultaneously. As a tie-breaking rule, we assume that in case of indifference, a rival does not enter.

At Stage 4 all rivals that entered and the incumbent compete à la Bertrand. All firms produce a homogenous good. Figure 1 summarizes the timing.

\begin{tabular}{cccc}
\hline $\mathbf{1}$ & & & \\
$\begin{array}{c}\text { Incumbent } \\
\text { invests }\end{array}$ & $\mathbf{2}\left(c_{1}, \ldots, c_{N}\right)$ & $\mathbf{3}$ Entry & $\mathbf{4}$ Bertrand \\
are drawn & decisions & competition
\end{tabular}

Figure 1: Timing

Consumers have unit demand and a willingness-to-pay of one. ${ }^{11}$ If there are two or more cheapest firms, they buy from the firm with the lower production costs. This assumption is solely made to avoid open set problems. So that the market is always served, we assume that $C<1$.

There is perfect information, and each firm maximizes its expected profit. Our solution concept is subgame perfect Nash equilibrium and we concentrate on pure strategy equilibria.

\section{ANALYSIS}

\subsection{BERTRAND COMPETITION AND ENTRY}

Standard analysis of the Bertrand game yields that rival $j$ 's profit when it entered the market is

$$
\left.\pi_{j}^{\text {entry }}\right|_{\mathcal{M}}=\max \left\{0, \min \left\{c_{i}\right\}_{i \in \mathcal{M} \backslash j}-c_{j}\right\}-Z,
$$

where $j \in\{1, \ldots, N\}$ and $\mathcal{M} \subseteq\{0, \ldots, N\}$ is the set of all firms which are in the market, that is, the incumbent firm 0 plus the rivals that entered. The first term of the formula is the Bertrand profit. If firm $j$ does not have the lowest production costs among all firms in the market, it makes

\footnotetext{
${ }^{11}$ That is, the incumbent's revenue is at most 1 . So any investment $k>1$ is dominated by $k=0$. So one could relax Assumption 1(iii) to $c_{0}(1)>Z$.
} 
a Bertrand profit of zero. Otherwise its Bertrand profit is the minimum per-unit cost of all other firms in the market minus its own production costs. The second term is cost of entry.

When a rival does not enter it makes zero profits. Hence, no rival wants to enter in equilibrium, if and only if

$$
\left.\pi_{j}^{\text {entry }}\right|_{\mathcal{M}=\{0\}} \leq 0 \quad \forall j \in\{1, \ldots, N\}
$$

With (1) this can be rewritten as

$$
c_{0}(k) \leq c_{j}+Z \quad \forall j \in\{1, \ldots, N\}
$$

When this condition does not hold, the equilibrium is such that some rival $j$ enters. Then $c_{0}(k)>c_{j}$ for sure, which implies that the incumbent will make a Bertrand profit of zero. Hence, the incumbent's profit is

$$
\pi_{0}(k)= \begin{cases}\pi_{0}^{\text {no entry }}(k)=1-c_{0}(k)-k & \text { for } c_{0}(k) \leq \underline{c}+Z \\ \pi_{0}^{\text {entry }}(k)=-k & \text { otherwise }\end{cases}
$$

where we defined $\underline{c}:=\min _{j \in\{1, \ldots, N\}}\left\{c_{j}\right\}$ as the minimum production costs of rivals.

\subsection{INVESTMENT}

The incumbent's expected profit when it invests $k$ is

$$
\mathbb{E}\left[\pi_{0}(k)\right]=\pi_{0}^{\text {no entry }}(k) \operatorname{Prob}^{\text {no entry }}(k)+\pi_{0}^{\text {entry }}(k) \operatorname{Prob}^{\text {entry }}(k) .
$$

Using (4) we can rewrite this as

$$
\mathbb{E}\left[\pi_{0}(k, F)\right]=\left[1-c_{0}(k)\right]\left[1-F\left(c_{0}(k)-Z\right)\right]-k,
$$

where $F$ is the distribution function from which $\underline{c}$ is drawn. The incumbent's incentives to invest are determined by the marginal effect of investment on its expected profit.

Consider first the benchmark case where entry never occurs (formally, where $F(C-Z)=0)$ :

$$
\frac{d \mathbb{E}\left[\pi_{0}(k, F)\right]}{d k}=-c_{0}^{\prime}(k)-1 .
$$


On the one hand, a higher investment $k$ increases the Bertrand profit by decreasing the production costs; this is captured by the first term on the right-hand side. On the other hand, a higher investment increases the investment costs; this is described by the second term.

Next, consider the more interesting case where entry may occur:

$$
\begin{aligned}
\frac{d \mathbb{E}\left[\pi_{0}(k, F)\right]}{d k}=-c_{0}^{\prime}(k)[1-F & \left.\left(c_{0}(k)-Z\right)\right] \\
& \left.-\left[1-c_{0}(k)\right] f\left(c_{0}(k)-Z\right)\right) c_{0}^{\prime}(k)-1,
\end{aligned}
$$

where $f$ is the density function which belongs to the distribution function $F$. What has changed through the entry threat? On the one hand, the return of investment is lower: it becomes less likely that the investment is actually used in production; see the first term. Put differently, it is less likely that the investment "pays off". This is called the Schumpeterian effect. ${ }^{12}$ On the other hand, the return of investment is higher: investing more makes entry less likely; see the second term. We call this the entry deterrence effect of investment. ${ }^{13}$ Since both effects run into different directions, it may well be the case that the incumbent's incentive to invest is higher with an entry threat than without one.

For concreteness, we assume that the production costs $\left(c_{1}, \ldots, c_{N}\right)$ of the rivals are independently drawn from exponential density functions. Rival $j$ 's costs are drawn from density

$$
f_{j}\left(c_{j}\right)=\lambda_{j} e^{-\lambda_{j} c_{j}}
$$

with $\lambda_{j}>0$ and corresponding distribution function

$$
F_{j}\left(c_{j}\right)=1-e^{-\lambda_{j} c_{j}} .
$$

The nice feature when all $c_{j}$ 's are independently and exponentially dis-

12 See also Aghion et al. (2001, 2005a). The Schumpeterian effect is closely related to the discouragement effect in Aghion et al. (2005b, 2009).

${ }^{13}$ Aghion and Griffith (2005) call this the Darwinian effect of competition. Our entry deterrence effect is similar to the escape-entry effect considered by Aghion et al. (2005b, 2009) and the escape-competition effect developed in Aghion et al. (2001, 2005a). 
tributed is that $\underline{c}$ is exponentially distributed, too: ${ }^{14}$

$$
F(\underline{c})=1-e^{-\lambda \underline{c}}, \text { with } \lambda:=\sum_{j=1}^{N} \lambda_{j} .
$$

Hence we can allow for heterogeneity of the rivals through different $\lambda_{j} \mathrm{~s}$ without complicating the analysis. The parameter $\lambda$ captures the strength of the entry threat. It increases with the number $N$ and the quality $\lambda_{j}$ of rivals. When $\lambda=0$ we say that there is no entry threat.

Under the exponential distribution, we get the incumbent's expected profit

$$
\mathbb{E}\left[\pi_{0}(k, \lambda)\right]=\left[1-c_{0}(k)\right] e^{-\lambda\left(c_{0}(k)-Z\right)}-k .
$$

Let the optimal investment be given by the function $k^{*}(\lambda)$.

P R O P O S I T O N 1: When there is no entry threat, the incumbent invests a positive amount: $k^{*}(0)=c_{0}^{\prime-1}(-1)>0$. When the entry threat is huge $(\lambda \rightarrow \infty)$, the incumbent does not invest. An investment of at least $\hat{k}$, where $\hat{k}$ is an arbitrary positive investment level, cannot be optimal when $\lambda$ is sufficiently high.

So the incumbent invests some positive amount when there is no entry threat. In contrast, when the threat is overwhelming, entry occurs for sure and the incumbent does not invest at all. Then the Schumpeterian effect dominates the entry deterrence effect. The intuition for the last point of Proposition 1 is as follows: when the entry threat is sufficiently large, entry is very likely, even when the incumbent invests $\hat{k}$ or more. So the incumbent invests an amount less than $\hat{k}$ to save investment costs.

Next we explore the question under which circumstances a higher entry threat increases the optimal investment.

P R O P O S I I O N 2: Suppose that $C<\frac{1+Z}{2}$. The optimal investment $k^{*}(\lambda)$ is increasing in $\lambda$ for $\lambda \rightarrow 0$.

Intuitively, when the initial production costs $C$ are low, the incumbent's monopoly profit is high. Then the incumbent invests more when

\footnotetext{
${ }^{14}$ Technically we need the distribution of the first-order statistics for our analysis.
} 
there is a small entry threat than when there is no threat, because it wants to defend its monopoly. Therefore, for low entry threats the entry deterrence effect dominates the Schumpeterian effect.

The next Proposition follows directly from Propositions 1 and 2 .

P R O P O S I T O N 3: Suppose that $C<\frac{1+Z}{2}$. The optimal investment $k^{*}(\lambda)$ is hump-shaped in $\lambda$.

To sum up, when the entry threat is small and increases, the incumbent invests more to make entry unlikely. This is due to the entry deterrence effect. However, when the threat becomes huge, entry can no longer profitably be made unlikely and the investment becomes small. Then the Schumpeterian effect dominates.

\section{ALTERNATIVE TIMING}

We now consider an alternative timing where Stage 1 and 2 are interchanged and show that our results stay robust. Suppose that the incumbent already knows $\left(c_{1}, \ldots, c_{N}\right)$ when deciding about investment. The optimal investment is denoted by $k^{* *}(\underline{c})$.

When the rivals' production costs are large, there is no entry threat; formally, $\underline{c} \geq C-Z$. Even when the incumbent does not invest, no rival would enter. From (7) we get that the incumbent's investment, which we denote by $k^{* *}(\infty)$ to make clear that $\underline{c}$ is large, is then

$$
k^{* *}(\infty)=c_{0}^{\prime-1}(-1)>0
$$

Observe that without an entry threat the incumbent's investment is the same in both timings.

The profit function, given that entry is deterred, is concave in $k$ :

$$
d^{2} \pi_{0}^{\text {no entry }}(k) / d k^{2}=-c_{0}^{\prime \prime}(k)<0
$$

So when the incumbent deters entry, it either invests $k^{* *}(\infty)$ or, if that is not enough, just enough to deter entry:

$$
k^{\text {deter entry }}(\underline{c})= \begin{cases}k^{* *}(\infty)=c_{0}^{-1}(-1) & \text { for } \underline{c} \geq c_{0}\left(k^{* *}(\infty)\right)-Z, \\ c_{0}^{-1}(\underline{c}+Z) & \text { otherwise. }\end{cases}
$$


When the incumbent does not want to deter entry, $\pi_{0}(k)=-k$, see (4). So the optimal investment is

$$
k^{\text {do not deter entry }}(\underline{c})=0 .
$$

This yields zero profits. ${ }^{15}$

Does the incumbent want to deter entry or not? Denote the investment above which entry deterrence yields a loss by $\bar{k}$. It is implicitly given by

$$
1-c_{0}(\bar{k})-\bar{k}=0 \text {. }
$$

Through the assumptions made before, existence and uniqueness are guaranteed. ${ }^{16}$

So when investing according to (15) yields an investment which is at most $\bar{k}$, it is optimal to deter entry and to follow this investment rule. Otherwise, not deterring entry and zero investments are optimal, see (16). The following lemma summarizes our findings. They are illustrated in Figure 2. ${ }^{17}$

L E M M A 1: When $\underline{c}$ is below $c_{0}(\bar{k})-Z$ the incumbent does not invest and entry occurs. Otherwise the incumbent invests according to (15) and entry is deterred.

Figure 2 shows a hump-shaped relationship between the incumbent's investment and $\underline{c}$. But to make the results comparable to the one yielded

${ }^{15}$ Note, even a zero investment may deter entry. So the previous equation is only sensible when entry occurs, given a zero investment.

${ }^{16} \bar{k}$ exists because $c_{0}(k)$ is continuous in $k, \pi_{0}^{\text {no entry }}(k=1)<0$, and $\pi_{0}^{\text {no entry }}(k=$ $0)>0$. Uniqueness follows from $\pi_{0}^{\text {no entry }}(k=0)>0$ and the concavity of $\pi_{0}^{\text {no entry }}(k)$ in $k$.

${ }^{17}$ The following properties of $k^{\text {deter entry }}(\underline{c})$ are useful to construct the Figure.

(i) $k^{\text {deter entry }}(\underline{c})$ is continuous at $\underline{c}=c_{0}\left(k^{* *}(\infty)\right)-Z$,

(ii) $k^{\text {deter entry }}(\underline{c})$ has a kink at $\underline{c}=c_{0}\left(k^{* *}(\infty)\right)-Z$ :

$$
\begin{aligned}
& \lim _{\underline{c} \searrow c_{0}\left(k^{* *}(\infty)\right)-Z} d k^{\text {deter entry }}(\underline{c}) / d \underline{c}=0 \text { and }
\end{aligned}
$$

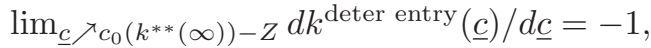

(iii) $k^{\text {deter entry }}(\underline{c})$ is constant in $\underline{c}$ for $\underline{c}>c_{0}\left(k^{* *}(\infty)\right)-Z$, and

(iv) $k^{\text {deter entry }}(\underline{c})$ is decreasing and convex in $\underline{c}$ for $\underline{c}<c_{0}\left(k^{* *}(\infty)\right)-Z$. 


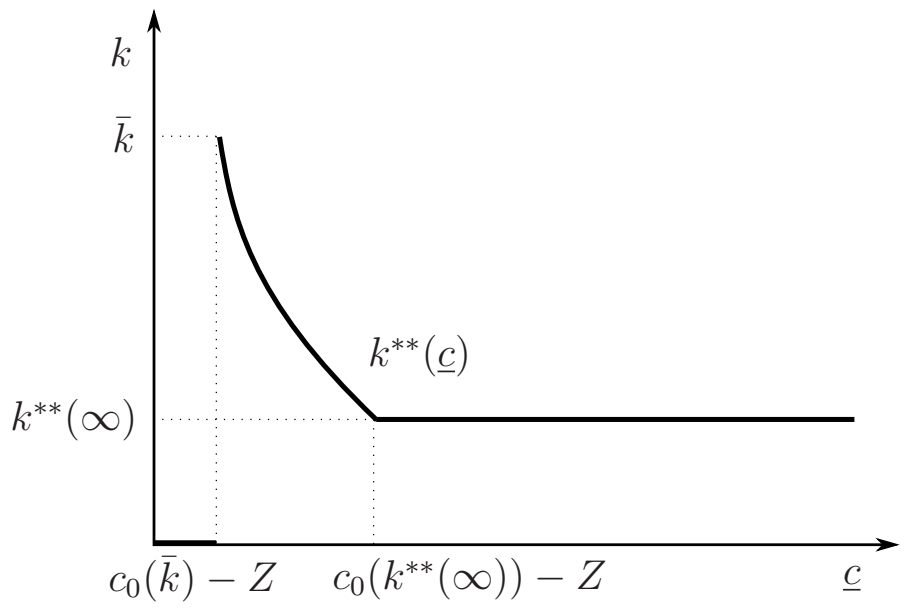

Figure 2: Incumbent's investment decision

under the original timing we seek to answer the following question: how large is the average investment of the incumbent, given $\lambda$ ? Again, we assume that rivals cost are drawn from an exponential density function.

P R O P O S I T I O N 4: $\lim _{\lambda \rightarrow 0} \mathbb{E}[k \mid \lambda]=k^{* *}(\infty), \lim _{\lambda \rightarrow \infty} \mathbb{E}[k \mid \lambda]=0$. If

$$
\frac{\left(c_{0}\left(k^{* *}(\infty)\right)-c_{0}(\bar{k})\right)^{2}}{2}-\left(c_{0}(\bar{k})-Z\right) k^{* *}(\infty)>0,
$$

then the maximum of $\mathbb{E}[k \mid \lambda]$ is greater than $k^{* *}(\infty)$.

Intuitively, when $\lambda \rightarrow 0$ the probability that $\underline{c}$ will lie in the right region of Figure 2 approaches one. Therefore, the incumbent's expected investment is $k^{* *}(\infty)$. In contrast, when $\lambda \rightarrow \infty$ the probability that $\underline{c}$ will lie in the left region of the Figure approaches one. Therefore, the incumbent's expected investment is zero.

The intuition for the sufficient condition is as follows: if $\lambda$ is low, it is very likely that $\underline{c}$ will lie in the right region of Figure 2. Then $\mathbb{E}[k \mid \lambda] \approx k^{* *}(\infty)$. When $\lambda$ increases, it becomes more likely that $\underline{c}$ is in the left or in the middle region of Figure 2. When $k^{* *}(\infty)$ is small enough, $\mathbb{E}[k \mid \lambda]$ increases with $\lambda$ for small $\lambda \mathrm{s}$. The reason is that the reduction of the probability to get a medium investment $k^{* *}(\infty)$ is overcompensated through an increased probability to get a high investment. ${ }^{18}$

${ }^{18} \mathrm{An}$ alternative interpretation is that $\bar{k}$ is sufficiently high. Leaving the derivatives 
The results with the alternative timing are qualitatively the same as with the original timing. For a low entry threat, the incumbent's average investment is moderate. For a medium threat, the incumbent's average investment is, under some conditions, relatively high. For a huge entry threat, the incumbent's expected investment approaches zero. Therefore, the relationship between the incumbent's investment and the entry threat is again hump-shaped.

\section{FURTHER ROBUSTNESS ISSUES}

\subsection{UNIFORM DISTRIBUTION}

Do our results also hold when $\underline{c}$ is not exponentially distributed? Because the intuition for our results does not depend on this assumption, we are quite confident that the results also hold with other-though certainly not for all-distributions. The robustness is easy to show for the uniform assumption. Suppose $\underline{c}$ is uniformly distributed between $\underline{u}$ and $\bar{u}$, with $\underline{u}<\bar{u}$. Fix $\bar{u}-\underline{u}$ and interpret a higher entry threat as a lower $\underline{u}$ or $\bar{u}$.

Consider the original timing. We will look at three different entry threats and show that the incumbent's optimal investment is highest for a mediocre entry threat. First, when $\underline{u}>C$ there is essentially no entry threat and the incumbent's investment is $k^{*}(0)=c_{0}^{\prime-1}(-1)>0$, see (7). Second, when $\underline{u}=c_{0}\left(k^{*}(0)\right)+Z-\epsilon$, where $\epsilon$ is small and positive, then there is an entry threat: investing $k^{*}(0)$ is not sufficient to deter entry in all cases. From (8) we see that the marginal effect of a higher investment on the incumbent's expected profit is positive at $k=k^{*}(0)$ : (i) the term $\left[1-F\left(c_{0}(k)-Z\right)\right]$ is almost 1 because of the small $\epsilon$ (i.e., the Schumpeterian effect is weak); (ii) $\left.\left[1-c_{0}(k)\right] f\left(c_{0}(k)-Z\right)\right) c_{0}^{\prime}(k)$ is substantial (i.e., the entry deterrence effect is strong). So the incumbent invests more than in the case $\underline{u}>C$. Third, when $\bar{u}$ is close to zero

of $c_{0}(k)$ unchanged, but shifting $c_{0}(0)=C$, the sufficient condition is easier met with a lower $C$. That is, we more likely get a hump-shaped relationship when the incumbent's initial costs are low so that it has a strong incentive to defend its monopoly. Note that also in the original timing we assumed that $C$ is sufficiently low; see Propositions 2 and 3. 
the incumbent cannot profitably deter entry (this is due to Assumption 1(iii)) and the optimal investment is zero.

Consider next the alternative timing. We again look at three different entry threats. First, as above, when $\underline{u}>C$ there is essentially no entry threat and the incumbent invests $k^{* *}(\infty)=c_{0}^{\prime-1}(-1)>0$. Second, when $\underline{u}$ is between $c_{0}(\bar{k})-Z$ and $c_{0}\left(k^{* *}(\infty)\right)-Z$ then the incumbent invests at least $k^{* *}(\infty)$ and sometimes strictly more. Third, when $\bar{u}$ is below $c_{0}(\bar{k})-Z$ then the incumbent will never invest. So again, the incumbent's optimal investment is highest for a mediocre entry threat.

Finally, observe that with both timings we do not need to make an assumption on the incumbent's initial costs. This is in contrast to the case with an exponential distribution of $\underline{c}$.

\subsection{ALTERNATIVE INTERPRETATIONS}

Our model can be interpreted and modified in several ways.

First, consider the model without stochasticity of the rivals' production costs. Figure 2 shows a hump-shaped relationship between the incumbent's investment and the production costs of the most efficient rival, $\underline{c}$. When we interpret $\underline{c}$ as the measure of the entry threat (a lower $\underline{c}$ is a greater threat), we again get the prediction that the relationship between the incumbent's investment and the entry threat is hump-shaped.

Second, it is easily verified that the model (with the original as well as with the alternative timing) stays equivalent when entry costs are stochastic and rivals' production costs are fixed. The key insight is that the rivals' entry decisions stay the same, see (3). Then a higher entry threat is interpreted as a draw of the entry costs from an exponential density with a lower mean. The reason for on average lower entry costs may be a weaker patent protection/property rights ${ }^{19}$ or lower costs of imitation.

Third, one can combine several of the robustness issues. For example, one can consider the model without stochasticity, fix the rivals' produc-

\footnotetext{
${ }^{19}$ When patent protection is weaker, it is easier or not necessary for rival firms to invent around the incumbent's patent.
} 
tion costs, and vary the entry costs. Then the entry costs can be taken as a measure of the entry threat. One again yields the prediction of a hump-shaped relationship between the incumbent's investment and the entry threat. Another example is to consider the model with stochastic entry costs which are uniformly distributed.

\section{HOW IMPORTANT ARE THE INCUMBENT'S INITIAL COSTS?}

One directly sees that the sufficient condition for having a hump-shaped relationship between the incumbent's $R \& D$ investment and the entry threat is easier to satisfy when the initial production costs $C$ are lower. This holds under both timings. But we can go beyond these results. Consider the original timing.

P R O P O S I T I O N 5: When there is an entry threat, the incumbent's optimal investment is decreasing in the initial costs $C$.

Observe that without an entry threat the incumbent's optimal investment is independent of $C$. Therefore, Proposition 5 implies that with high initial costs it is more likely that an entry threat (and possibly every threat) decreases the incumbent's optimal investment. This is similar to the finding of Aghion et al. (2005b, 2009) for an incumbent which is further behind the frontier. ${ }^{20}$ The intuition for our result is as follows. The Schumpeterian effect is more likely to dominate the entry deterrence effect when the incumbent has initially high costs, because high costs make entry deterrence (i) less profitable, since the production costs are relatively high and (ii) more difficult, since for a given investment entry becomes more likely.

With the alternative timing, similar arguments hold. Without an entry threat, the incumbent's optimal investment is independent of $C$. When there is an entry threat, a higher $C$ leads to lower expected investments because the range where the incumbent does not invest at all increases and the maximal investment $\bar{k}$ decreases.

\footnotetext{
${ }^{20}$ We have no technological frontier in our model. But closeness to the frontier corresponds best to low initial production costs $C$.
} 


\section{CONCLUSIONS}

The model formalizes the idea that an incumbent rests on its laurels when there is no threat, fights when the threat is mediocre, and gives up when the threat is huge. We measure the threat by the number and quality of rival firms which may enter the market. A higher threat may motivate an incumbent to invest more in R\&D to deter entry or to make it unlikely; then the entry deterrence effect dominates. However, when the threat is overwhelming, the incumbent has little chance to deter entry and invests little or not at all; then the Schumpeterian effect dominates. Therefore, the relationship between the incumbent's investment and the entry threat is hump-shaped.

\section{APPENDIX}

\subsection{PROOF OF PROPOSITION 1}

The derivative of (12) is

$$
\frac{d \mathbb{E}\left[\pi_{0}(k, \lambda)\right]}{d k}=-c_{0}^{\prime}(k) e^{-\lambda\left(c_{0}(k)-Z\right)}\left(1+\lambda\left[1-c_{0}(k)\right]\right)-1 .
$$

First consider the case of no entry threat, $\lambda=0$. Then

$$
d \mathbb{E}\left[\pi_{0}(k, 0)\right] / d k=-c_{0}^{\prime}(k)-1 .
$$

The optimal investment $k^{*}(0)$ solves $c_{0}^{\prime}\left(k^{*}(0)\right)=-1$. Hence, $k^{*}(0)=$ $c_{0}^{\prime-1}(-1)$, which is positive through Assumption 1(iv).

Next, consider the other extreme of $\lambda \rightarrow \infty$. Then Assumption 1(iii) implies that entry occurs for sure for all investment levels $k$. So

$\mathbb{E}\left[\pi_{0}(k, \lambda)\right]=-k$ for all $k$. Therefore the incumbent chooses not to invest. Formally, $k^{*}(\infty)=0$.

Finally, we prove the last part of Proposition 1. By Assumption 1 (iii) and $Z>0$ we have $c_{0}(k)>0 \forall k$. Moreover, the expected revenue is at most 1 . Hence, $k \geq 1$ leads to a loss for the incumbent. This is dominated by $k=0$, which yields a non-negative profit. Therefore, an investment of $k \geq 1$ can never be optimal. 
From (12) we get that $\forall k \in[\hat{k}, 1]$ we have

$$
\begin{aligned}
\mathbb{E}\left[\pi_{0}(k, \lambda)\right] & =\left[1-c_{0}(k)\right] e^{-\lambda\left(c_{0}(k)-Z\right)}-k \\
& \leq\left(1-c_{0}(1)\right) e^{-\lambda\left(c_{0}(1)-Z\right)}-k \\
& \leq\left(1-c_{0}(1)\right) e^{-\lambda\left(c_{0}(1)-Z\right)}-\hat{k}
\end{aligned}
$$

When $\lambda$ is sufficiently high, the last line is negative:

$$
\left(1-c_{0}(1)\right) e^{-\lambda\left(c_{0}(1)-Z\right)}-\hat{k}<0
$$

which proves that

$$
\mathbb{E}\left[\pi_{0}(k, \lambda)\right]<0 \quad \forall k \in[\hat{k}, 1]
$$

Note that investing nothing yields a non negative expected profit. Hence, investing $k \geq \hat{k}$ is dominated by $k=0$ and cannot be optimal.

\subsection{PROOF OF PROPOSITION 2}

We look at the effect of $\lambda$ on the marginal return of investment:

$$
\begin{aligned}
& \frac{d^{2} \mathbb{E}\left[\pi_{0}(k, \lambda)\right]}{d k d \lambda}=\quad c_{0}^{\prime}(k) e^{-\lambda\left(c_{0}(k)-Z\right)}\left(c_{0}(k)-Z\right) \\
& -\left[1-c_{0}(k)\right] e^{-\lambda\left(c_{0}(k)-Z\right)} c_{0}^{\prime}(k) \\
& +\left[1-c_{0}(k)\right] e^{-\lambda\left(c_{0}(k)-Z\right)} \lambda c_{0}^{\prime}(k)\left(c_{0}(k)-Z\right) \text {. }
\end{aligned}
$$

The first term on the RHS is negative: a higher $\lambda$ increases the probability of entry. That is, the return on investment decreases due to the Schumpeterian effect. The remaining terms capture the entry deterrence effect. The sign of the sum of the remaining terms is ambiguous. That is, entry deterrence may or may not become more attractive when $\lambda$ increases. Given some $k$, when $\lambda$ is small (large), the remaining terms are positive (negative). Since we seek to explore whether it is possible that a higher entry threat increases the optimal investment, we consider the case $\lambda \rightarrow 0$ :

$$
\lim _{\lambda \rightarrow 0} \frac{d^{2} \mathbb{E}\left[\pi_{0}(k, \lambda)\right]}{d k d \lambda}=c_{0}^{\prime}(k)\left(c_{0}(k)-Z\right)-\left[1-c_{0}(k)\right] c_{0}^{\prime}(k),
$$

which has the same sign as

$$
1-2 c_{0}(k)+Z
$$


So when

$$
c_{0}(k)<\frac{1+Z}{2}
$$

we have that $\lim _{\lambda \rightarrow 0} d^{2} \mathbb{E}\left[\pi_{0}(k, \lambda)\right] / d k d \lambda>0$. When

$$
C<\frac{1+Z}{2}
$$

this is true for all $k$. Suppose that this is true. Then also for small $\lambda \mathrm{s}$ we have $d^{2} \mathbb{E}\left[\pi_{0}(k, \lambda)\right] / d k d \lambda>0$.

First note that $k^{*}(0)$ is unique and given by the first-order condition $d \mathbb{E}\left[\pi_{0}\left(k^{*}(0), 0\right)\right] / d k \stackrel{!}{=} 0$. Hence, because $d^{2} \mathbb{E}\left[\pi_{0}(k, \lambda)\right] / d k d \lambda>0$ for small $\lambda$ s we must have $k^{*}(\lambda) \neq k^{*}(0)$.

Second, by the optimality of $k^{*}(\lambda)$ and $k^{*}(0)$ we have

$$
\begin{aligned}
& \mathbb{E}\left[\pi_{0}\left(k^{*}(\lambda), \lambda\right)\right] \geq \mathbb{E}\left[\pi_{0}\left(k^{*}(0), \lambda\right)\right], \\
& \mathbb{E}\left[\pi_{0}\left(k^{*}(0), 0\right)\right] \geq \mathbb{E}\left[\pi_{0}\left(k^{*}(\lambda), 0\right)\right]
\end{aligned}
$$

which can be transformed to

$$
\begin{gathered}
\int_{k^{*}(0)}^{k^{*}(\lambda)} \frac{\partial \mathbb{E}\left[\pi_{0}(k, \lambda)\right]}{\partial k} d k \geq 0 \\
\int_{k^{*}(\lambda)}^{k^{*}(0)} \frac{\partial \mathbb{E}\left[\pi_{0}(k, 0)\right]}{\partial k} d k \geq 0 .
\end{gathered}
$$

Third, because of $d^{2} \mathbb{E}\left[\pi_{0}(k, \lambda)\right] / d k d \lambda>0$ for small $\lambda$ s we have

$$
\frac{\partial \mathbb{E}\left[\pi_{0}(k, \lambda)\right]}{\partial k}>\frac{\partial \mathbb{E}\left[\pi_{0}(k, 0)\right]}{\partial k} .
$$

Fourth, because of (32) the inequalities (30) and (31) cannot be satisfied for $k^{*}(\lambda)<k^{*}(0)$. Hence, because an optimum exists, and from before we know that $k^{*}(\lambda) \neq k^{*}(0)$ it must hold that $k^{*}(\lambda)>k^{*}(0)$ for small $\lambda \mathrm{s}$.

\subsection{PROOF OF PROPOSITION 4}

With help of Lemma 1, we yield that the incumbent's expected investment is

$$
\begin{aligned}
\mathbb{E}[k \mid \lambda]= & \int_{0}^{c_{0}(\bar{k})-Z} 0 d F(\underline{c})+\int_{c_{0}(\bar{k})-Z}^{c_{0}\left(k^{* *}(\infty)\right)-Z} k^{* *}(\cdot) d F(\underline{c}) \\
& +\int_{c_{0}\left(k^{* *}(\infty)\right)-Z}^{\infty} k^{* *}(\infty) d F(\underline{c}),
\end{aligned}
$$


where $k^{* *}(\infty)=c_{0}^{\prime-1}(-1)$ and $k^{* *}(\cdot)=c_{0}^{-1}(Z+\underline{c})$ for the second integral, see (15). All three integrals always have a positive probability mass because Assumption 1(iii) implies that $c_{0}(\bar{k})-Z>0$.

The analysis when $\lambda \rightarrow 0$ or $\lambda \rightarrow \infty$ is especially easy. When $\lambda \rightarrow 0$, the probability that $\underline{c}$ is so high that the incumbent will invest $k^{* *}(\infty)$ approaches one. Moreover, for other levels of $\underline{c}$, the investments are in the interval $[0, \bar{k}]$. Hence, $\lim _{\lambda \rightarrow 0} \mathbb{E}[k \mid \lambda]=k^{* *}(\infty)$. When $\lambda \rightarrow \infty$, the probability that $\underline{c}$ is in the region where the incumbent does not invest approaches one and $\lim _{\lambda \rightarrow \infty} \mathbb{E}[k \mid \lambda]=0$.

But how large is $\mathbb{E}[k \mid \lambda]$ if we have a medium $\lambda$ value? Since all areas have a positive weight, $k^{* *}(\infty)$ as well as $k^{* *}(\cdot)$ are non-negative and at most $\bar{k}$, it follows that $\bar{k}>\mathbb{E}[k \mid \lambda]>0$. But under what conditions can $\mathbb{E}[k \mid \lambda]$ exceed $k^{* *}(\infty)$ ?

For medium values of $\underline{c}$, i.e., when $c_{0}(\bar{k})-Z<\underline{c}<c_{0}\left(k^{* *}(\infty)\right)-Z$, the optimal investment is given by $c_{0}^{-1}(Z+\underline{c})$. Denote this part of the investment function by $k_{\text {medium }}^{* *}(\cdot)$. The first-order Taylor approximation of $k_{\text {medium }}^{* *}(\cdot)$ is

$$
k_{\text {medium }}^{* *}(\cdot) \approx k^{* *}(\infty)+c_{0}\left(k^{* *}(\infty)\right)-Z-\underline{c} .
$$

Since $d^{2} k_{\text {medium }}^{* *}(\cdot) / d \underline{c}^{2}>0$ (see previous analysis) we do not overestimate $k_{\text {medium }}^{* *}(\cdot)$ by this approximation. Next, we put the approximation of $k_{\text {medium }}^{* *}(\cdot)$ into $(33)$, so

$$
\begin{aligned}
\mathbb{E}[k \mid \lambda] \geq \quad & \int_{0}^{c_{0}(\bar{k})-Z} 0 d F(\underline{c}) \\
& +\int_{c_{0}(\bar{k})-Z}^{c_{0}\left(k^{* *}(\infty)\right)-Z}\left(k^{* *}(\infty)+c_{0}\left(k^{* *}(\infty)\right)-Z-\underline{c}\right) d F(\underline{c}) \\
& +\int_{c_{0}\left(k^{* *}(\infty)\right)-Z}^{\infty} k^{* *}(\infty) d F(\underline{c}) .
\end{aligned}
$$

For the exponential density function, this can be rewritten as

$$
\begin{aligned}
\mathbb{E}[k \mid \lambda] \geq \quad & \int_{c_{0}(\bar{k})-Z}^{c_{0}\left(k^{* *}(\infty)\right)-Z}\left[c_{0}\left(k^{* *}(\infty)\right)-Z-\underline{c}\right] \lambda e^{-\lambda \underline{c}} d \underline{c} \\
& +\int_{c_{0}(\bar{k})-Z}^{\infty} k^{* *}(\infty) \lambda e^{-\lambda \underline{c}} d \underline{c} .
\end{aligned}
$$


Since the exponential density is decreasing in $\underline{c}$ and the square bracket in the integral is linear in $\underline{c}$, an approximation and a lower bound of the first integral of (36) is

$$
\begin{aligned}
\int_{c_{0}(\bar{k})-Z}^{c_{0}\left(k^{* *}(\infty)\right)-Z} \frac{\left(c_{0}\left(k^{* *}(\infty)\right)-Z-c_{0}\left(k^{* *}(\infty)\right)+Z\right)+\left(c_{0}\left(k^{* *}(\infty)\right)-Z-c_{0}(\bar{k})+Z\right)}{2} \lambda e^{-\lambda \underline{c}} d \underline{\underline{c}} \\
=\int_{c_{0}(\bar{k})-Z}^{c_{0}\left(k^{* *}(\infty)\right)-Z} \frac{c_{0}\left(k^{* *}(\infty)\right)-c_{0}(\bar{k})}{2} \lambda e^{-\lambda \underline{c}} d \underline{c} . \quad \text { (37) }
\end{aligned}
$$

With (36) we get

$$
\begin{aligned}
\mathbb{E}[k \mid \lambda] \geq \quad & \frac{c_{0}\left(k^{* *}(\infty)\right)-c_{0}(\bar{k})}{2}\left[e^{-\lambda\left(c_{0}(\bar{k})-Z\right)}-e^{-\lambda\left(c_{0}\left(k^{* *}(\infty)\right)-Z\right)}\right] \\
& +k^{* *}(\infty) e^{-\lambda\left(c_{0}(\bar{k})-Z\right)}=: \Omega(\lambda)
\end{aligned}
$$

The derivative is

$$
\begin{aligned}
\frac{d \Omega(\lambda)}{d \lambda} e^{\lambda\left(c_{0}(\bar{k})-Z\right)}=\frac{c_{0}\left(k^{* *}(\infty)\right)-c_{0}(\bar{k})}{2} & \\
{\left[-\left(c_{0}(\bar{k})-Z\right)+\left(c_{0}\left(k^{* *}(\infty)\right)-Z\right) e^{-\lambda\left(c_{0}\left(k^{* *}(\infty)\right)-c_{0}(\bar{k})\right)}\right] } & -\left(c_{0}(\bar{k})-Z\right) k^{* *}(\infty) .
\end{aligned}
$$

Because $e^{\lambda\left(c_{0}(\bar{k})-Z\right)}>0$ the LHS has the same sign as $d \Omega(\lambda) / d \lambda$. Observe that

$$
\lim _{\lambda \rightarrow 0} \operatorname{RHS}>0 \Longleftrightarrow \frac{\left(c_{0}\left(k^{* *}(\infty)\right)-c_{0}(\bar{k})\right)^{2}}{2}-\left(c_{0}(\bar{k})-Z\right) k^{* *}(\infty)>0 .
$$

When this condition holds, $\Omega(\lambda)$ is increasing in $\lambda$ when $\lambda$ is sufficiently small. Because $\lim _{\lambda \rightarrow 0} \Omega(\lambda)=k^{* *}(\infty)$ then the maximum of $\Omega(\lambda)$ is greater than $k^{* *}(\infty)$. Because $\mathbb{E}[k \mid \lambda] \geq \Omega(\lambda)$, then also the maximum of $\mathbb{E}[k \mid \lambda]$ is greater than $k^{* *}(\infty)$.

\subsection{PROOF OF PROPOSITION 5}

As can be seen from (8),

$$
\left.\left.\frac{d^{2} \mathbb{E}\left[\pi_{0}(k, F)\right]}{d k d C}=2 c_{0}^{\prime}(k) f\left(c_{0}(k)-Z\right)\right)-\left(1-c_{0}(k)\right) c_{0}^{\prime}(k) f^{\prime}\left(c_{0}(k)-Z\right)\right)
$$

which is negative under the exponential distribution. A result which we will use below. 
Let the function $k^{* * *}(C \mid \lambda)$ denote the optimal investment depending on incumbent's initial costs $C$, given some $\lambda$. We next prove by contradiction. Let $C^{I}<C^{I I}$ and suppose that $k^{* * *}\left(C^{I I} \mid \lambda\right) \geq k^{* * *}\left(C^{I} \mid \lambda\right)$. One can rewrite the incumbent's expected profit as

$$
\begin{aligned}
\mathbb{E}\left[\pi_{0}\left(k^{* * *}\left(C^{I I} \mid \lambda\right) \mid C^{I I}\right)\right] & \\
& =\mathbb{E}\left[\pi_{0}\left(k^{* * *}\left(C^{I} \mid \lambda\right) \mid C^{I I}\right)\right]+\int_{k^{* * *}\left(C^{I} \mid \lambda\right)}^{k^{* * *}\left(C^{I I} \mid \lambda\right)} \frac{\partial \mathbb{E}\left[\pi_{0}\left(k \mid C^{I I}\right)\right]}{\partial k} d k .
\end{aligned}
$$

The optimality of $k^{* * *}\left(C^{I I} \mid \lambda\right)$ requires that the term with the integral is non-negative. The optimality of $k^{* * *}\left(C^{I} \mid \lambda\right)$ requires that

$$
\mathbb{E}\left[\pi_{0}\left(k^{* * *}\left(C^{I} \mid \lambda\right) \mid C^{I}\right)\right] \geq \mathbb{E}\left[\pi_{0}\left(k^{* * *}\left(C^{I I} \mid \lambda\right) \mid C^{I}\right)\right],
$$

or rewritten that

$$
\int_{k^{* * *}\left(C^{I} \mid \lambda\right)}^{k^{* * *}\left(C^{I I} \mid \lambda\right)} \frac{\partial \mathbb{E}\left[\pi_{0}\left(k, C^{I}\right)\right]}{\partial k} d k \leq 0 .
$$

But since $k^{* * *}\left(C^{I I} \mid \lambda\right) \geq k^{* * *}\left(C^{I} \mid \lambda\right), C^{I}<C^{I I}$, and $d^{2} \mathbb{E}\left[\pi_{0}(k, F)\right] / d k d C<$ 0 , it follows that

$$
\int_{k^{* * *}\left(C^{I} \mid \lambda\right)}^{k^{* * *}\left(C^{I I} \mid \lambda\right)} \frac{\partial \mathbb{E}\left[\pi_{0}\left(k, C^{I}\right)\right]}{\partial k} d k>\int_{k^{* * *}\left(C^{I} \mid \lambda\right)}^{k^{* * *}\left(C^{I I} \mid \lambda\right)} \frac{\partial \mathbb{E}\left[\pi_{0}\left(k, C^{I I}\right)\right]}{\partial k} d k,
$$

which is a contradiction.

Observe that this result does not require that $\underline{c}$ is exponentially distributed. We only used $d^{2} \mathbb{E}\left[\pi_{0}(k, F)\right] / d k d C<0$, which also holds with other distributions.

\section{LITERATURE}

Aghion, Philippe, Nick Bloom, Richard Blundell, Rachel Griffith, and Peter Howitt (2005a). Competition and Innovation: An Inverted-U Relationship. Quarterly Journal of Economics 120, 701-728.

Aghion, Philippe, Richard Blundell, Rachel Griffith, Peter Howitt, and Susanne Prantl (2009). The Effects of Entry on Incumbent Innovation and Productivity. Review of Economics and Statistics 91, 20-32.

Aghion, Philippe, Robin Burgess, Stephen J. Redding, and Fabrizio Zilibotti (2005b). 
Entry Liberalization and Inequality in Industrial Performance. Journal of the European Economic Association 3, 291-302.

Aghion, Philippe, Robin Burgess, Stephen J. Redding, and Fabrizio Zilibotti (2008). The Unequal Effects of Liberalization: Evidence from Dismantling the License Raj in India. American Economic Review 98, 1397-1412.

Aghion, Philippe and Rachel Griffith (2005). Competition and Growth. Reconciling Theory and Evidence. Cambridge: MIT Press.

Aghion, Philippe, Christopher Harris, Peter Howitt, and John Vickers (2001). Competition, Imitation and Growth with Step-by-Step Innovation. Review of Economic Studies 68, 467-492.

Aghion, Philippe and Peter Howitt (2006). Appropriate Growth Policy: A Unifying Framework. Journal of the European Economic Association 4, 269-314.

Aitken, Brian J. and Ann E. Harrison (1999). Do Domestic Firms Benefit from Direct Foreign Investment? Evidence from Venezuela. American Economic Review 89, 605-618.

Belleflamme, Paul and Cecilia Vergari (2006). Incentives to Innovate in Oligopolies. CORE Discussion Paper 2006/14.

Bessen, James E. and Eric Maskin (2009). Sequential Innovation, Patents, and Imitation. RAND Journal of Economics 40, 611-635.

Bessen, James E. and Michael J. Meurer (2008). Do Patents Perform Like Property? Boston University School of Law Working Paper 08-08.

Boldrin, Michele and David K. Levine (2009). A Model of Discovery. American Economic Review: Papers 85 Proceedings 99, 337-342.

Delbono, Flavio and Vincenzo Denicolo (1991). Incentives to Innovate in a Cournot Oligopoly. Quarterly Journal of Economics 106, 951-961.

Denicolo, Vincenzo and Piercarlo Zanchettin (2008). Competition, Market Selection and Growth. Working Paper.

Gallini, Nancy T. (1992). Patent policy and costly imitation. RAND Journal of Economics 23 52-63.

Gilbert, Richard J. (2006). Competition and Innovation. Journal of Industrial Organization Education 1, 1-23.

Gilbert, Richard J. and Steven C. Sunshine (1995). Incorporating Dynamic Efficiency Concerns in Merger Analysis: The use of Innovation Markets. Antitrust Law Journal $62,569-601$.

Goolsbee, Austan and Chad Syverson (2008). How do Incumbents Respond to the Threat of Entry? Evidence from the Major Airlines. Quarterly Journal of Economics 123, 1611-1633.

Javorcik, Beata Smarzynska (2004). Does Foreign Direct Investment Increase the 
Productivity of Domestic Firms? In Search of Spillovers Through Backward Linkages. American Economic Review 94, 605-627.

Lee, Tom and Louis L. Wilde (1980). Market Structure and Innovation: A Reformulation. Quarterly Journal of Economics 94, 429-436.

Lelarge, Claire and Benjamin Nefussi (2008). Exposure to Low-Wage Competition, Activity Changes and Quality Upgrading: An Empirical Assessment. Mimeo.

Lerner, Josh (2009). The Empirical Impact of Intellectual Property Rights on Innovation: Puzzles and Clues. American Economic Review Papers 85 Proceedings 99, 343-348.

MacDonald, James M. (1994). Does Import Competition Force Efficient Production? Review of Economics and Statistics 76, 721-727.

Mokyr, Joel (2009). Intellectual Property Rights, the Industrial Revolution, and the Beginnings of Modern Economic Growth. American Economic Review Papers 83 Proceedings 99, 349-355.

Pavcnik, Nina (2002). Trade Liberalization, Exit, and Productivity Improvements: Evidence from Chilean Plants. Review of Economic Studies 69, 245-276.

Sacco, Dario and Armin Schmutzler (2007). The Effects of Competition in Investment Games. University of Zurich Working Paper No. 0608.

Schmutzler, Armin (2007). The Relationship Between Competition and Innovation Why is it Such a Mess? University of Zurich Working Paper No. 0716.

Scotchmer, Suzanne (2004). Innovation and Incentives. Cambridge: MIT Press.

Segal, Ilya and Michael D. Whinston (2007). Antitrust in Innovative Industries. American Economic Review 97, 1703-1730.

Sutton, John (1990). Explaining Everything, Explaining Nothing? European Economic Review 34, 505-512.

Vickers, John (2009). Competition Policy and Property Rights. Discussion Paper University of Oxford, Department of Economics.

Vives, Xavier (2008). Innovation and Competitive Pressure. Journal of Industrial Economics 61, 419-469. 